\title{
Fuerzas Armadas y medios de comunicación: la mujer como elemento modernizador
}

\author{
Armed Forces and mass media: Women \\ as a modernizing factor
}

\author{
José-Manuel Moreno-Mercado; José-Manuel Jiménez-Cabello
}

Cómo citar este artículo:

Moreno-Mercado, José-Manuel; Jiménez-Cabello, José-Manuel (2019). “Fuerzas Armadas y medios de comunicación: la mujer como elemento modernizador". El profesional de la información, v. 28, n. 3, e280307.

https://doi.org/10.3145/epi.2019.may.07

Artículo recibido el 09-11-2018

Aceptación definitiva: 08-02-2019

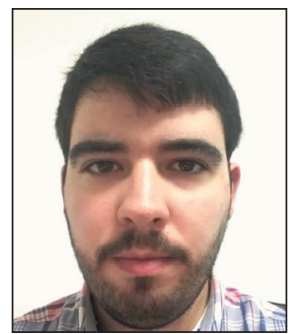

José-Manuel Moreno-Mercado https://orcid.org/0000-0003-0067-5051

Universidad de Granada

Facultad de Ciencias Políticas y

Sociología

Rector López Argüeta, s/n.

18001 Granada, España

jmmoreno95@correo.ugr.es $₫$

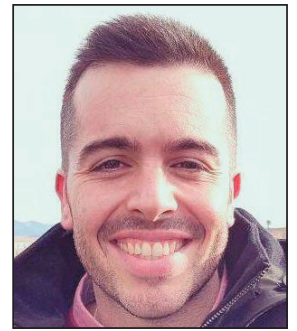

José-Manuel Jiménez-Cabello https://orcid.org/0000-0001-7518-2134

Universidad de Granada

Facultad de Ciencias Políticas y

Sociología

Rector López Argüeta, s/n.

18001 Granada, España

josejicabello@ugr.es

\section{Resumen}

Una eficiente interrelación entre Fuerzas Armadas, medios de comunicación y opinión pública es crucial para la elaboración de unas correctas políticas de seguridad y defensa. A pesar de que encontramos múltiples investigaciones sobre medios de comunicación y cuestiones de defensa nacional, los estudios que tratan esta temática desde el enfoque particular de las Fuerzas Armadas son prácticamente inexistentes en España. La presente investigación ahonda en los encuadres periodísticos utilizados por la prensa española a la hora de tratar las Fuerzas Armadas. A partir del análisis comparativo de dos períodos, esta investigación aporta datos novedosos sobre el tratamiento de la mujer dentro de las Fuerzas Armadas como elemento modernizador.

\section{Palabras clave}

Fuerzas Armadas; Encuadres; Medios de comunicación; Prensa digital; Diarios digitales; Cibermedios; Modernización; Mujer; Mujeres; Estudios de género; España.

\begin{abstract}
An efficient relationship between the Armed Forces, the media and the public opinion is crucial for an effective elaboration of security and defense policies. Despite there are available multiple researches about media and national defense, studies focused on the Armed Forces are practically non existent in Spain. The present research tries to deepen in the media frames used by the Spanish press when dealing with the armed forces. From a comparative analysis of two periods of time, this paper brings new data to this academic field about the treatment of women in the Armed Forces as a modernizing element.
\end{abstract}

\section{Keywords}

Armed Forces; Frames; Mass media; Digital media; Digital newspapers; Cybermedia; Online newspapers; Modernization; Woman; Women; Gender studies; Spain. 


\section{Introducción y aspectos teóricos}

Desde principios del siglo XX la mujer ha ido experimentado una progresiva integración en los ámbitos políticos e institucionales (León, 2001). En comunicación política las investigaciones han centrado sus esfuerzos en estudiar el lenguaje, la desigualdad social y la violencia contra las mujeres (Telcan-Reyes, 2018). Los estudios de género han aportado una abundante bibliografía académica en múltiples entornos científicos, en los que evidentemente se halla la comunicación. A pesar de que podemos encontrar gran número de estudios que abordan la inclusión de la mujer dentro de las instituciones públicas (Berríos, 2018) y su repercusión mediática ante sucesos políticos (Nicolini; Hansen, 2018), los trabajos que estudian el papel de la mujer dentro de las Fuerzas Armadas (FAS) españolas son prácticamente inexistentes.

La feminización de las FAS españolas no es un objeto de estudio de reciente origen; prueba de ello son algunas contribuciones clásicas como las de Fernández-Vargas (1997) u Hombrados, Olmedo y Val (2007). En esta línea podemos argumentar que la incorporación de la mujer en la institución castrense ha sido estudiada desde la sociología (Agudo-Arroyo, 2014), la seguridad y defensa (Solana-Cortés, 2009), y más cercanos al campo de la comunicación, desde la opinión pública en los casos de Díez-Nicolás (1986) o Ricoy-Casas (2006).

La inexistencia de estudios que analicen cómo los medios de comunicación en España han tratado la progresiva feminización de las Fuerzas Armadas dificulta la realización de un marco teórico solvente con obras de referencia. Resulta interesante ver cómo los medios, y más concretamente la prensa, plantean la temática de una institución con escasa repercusión pública, como indican los estudios del Centro de Investigaciones Sociológicas (CIS), y tradicionalmente considerada arcaica y conservadora (Pizarroso-Quintero, 2000).

\section{Los últimos estudios del CIS muestran una opinión pública favorable a las Fuerzas Armadas pero escaso interés en esta cuestión}

Dentro de la relación Fuerzas Armadas - medios de comunicación encontramos una extensísima bibliografía referente a cómo los medios han cubierto los sucesos propios de la política exterior, con especial énfasis en los conflictos armados (García-Marín, 2011; Rodríguez-Esperanza; Humanes, 2017; DeCillia, 2018). El número de investigaciones que analizan la relación entre la institución militar y los medios de comunicación es tan reducido que se limita a los modelos teóricos de la comunicación política en política exterior (Bennett, 1990; Entman, 2004) y a cuestiones de seguridad (Jordán; Torres, 2004).

La necesidad de acometer este tema desde el enfoque metodológico del framing se justifica desde el propio avance de la comunicación política y la cobertura mediática de la mujer en cuestiones de defensa y política exterior. El rol de la mujer en distintas áreas sociales tratadas por la prensa y la feminización de las editoriales periodísticas está arrojando estudios muy interesantes sobre relaciones públicas (Lambert, 2018), deportes (Cooky, 2018) o violencia sexual en ámbitos militares (Kuhl et al., 2018), entre otros muchos, pero dejando inexplorado el caso español.

El análisis y disección de encuadres han ocupado las investigaciones sobre comunicación política de los últimos años. El framing tiene como finalidad explicar cómo unos aspectos y consideraciones se destacan sobre otros dentro de un debate político (Bartholomé; Lecheler;

De-Vreese, 2018), de ahí su importancia. Partiendo de la base de que lo conflictivo es una de las pautas principales de la noticia, y que el conflicto de ideas es la columna vertebral de la democracia (Schattschneider, 1975), la manera en que la información se expone a la audiencia determinará sin duda ciertos juicios, valoraciones, decisiones y comportamientos que ésta realiza. Como señala Ardèvol-Abreu, los encuadres

"lejos de situarse exclusivamente en el emisor del mensaje, están localizados tanto en el emisor como en el receptor, el texto (informativo) y la cultura” (Ardèvol-Abreu, 2015, p. 425).

La identificación de encuadres, ya sea en el caso de los conflictos armados u otros sucesos, ha permitido establecer listados amplios y generalizables que han posibilitado inferir datos concluyentes sobre los contenidos informativos de los medios, así como el impacto que tienen sobre la audiencia que los consume (Muñiz, 2011).

La utilización de encuadres periodísticos conforma la columna vertebral de la presente investigación. El incipiente estudio del tratamiento mediático de las mujeres que integran las Fuerzas Armadas españolas implica ciertas limitaciones, como puede ser una muestra de análisis reducida. Sin embargo esta investigación pretende contribuir con un acercamiento genérico, pero no menos exhaustivo, al conocimiento de las prácticas periodísticas que se ofrecen y se han ofrecido por la prensa en España sobre la mujer en el ejército.

\section{Metodología}

El objetivo principal de este estudio consiste en identificar los encuadres presentes en los diarios españoles El país, El mundo y La vanguardia cuando realizan la cobertura sobre personal femenino en las Fuerzas Armadas españolas y si dichos encuadres han evolucionado durante los últimos años. Partiendo de la base de que la integración de la mujer 
se ha realizado de forma progresiva en todos los estamentos de la sociedad y las instituciones públicas, se plantea la siguiente hipótesis:

H1: La cobertura mediática sobre las mujeres militares ha evolucionado durante los últimos años presentándose en la actualidad como un elemento modernizador.

Se han planteado los siguientes objetivos específicos:

- Seleccionar tres diarios españoles de gran tirada nacional y líneas ideológicas divergentes. La elección de la prensa por encima de otros medios de comunicación se debe a su importancia dentro de la sociedad (Vicent, 2000).

- Obtener los editoriales a partir de la base de datos MyNews online a través de sus monitores de búsqueda con operadores booleanos.

- Analizar una muestra que, aunque pequeña debido a su escasa cobertura ( $n=173$ ), englobe dos períodos temporales amplios (2017-2018 y 2012-2013) ya que en nuestra hipótesis planteamos que dicha cobertura ha experimentado variaciones.

- Medir la presencia de los encuadres a partir de la elaboración de 10 preguntas dicotómicas (presencia/ausencia) siguiendo el esquema propuesto por Entman (1993).

Tras la revisión bibliográfica presentada, y debido a la inexistencia de trabajos en esta línea, se espera encontrar la presencia de 4 encuadres a partir de una primera aproximación exploratoria:

- Política: debido a la indudable interrelación con el poder político, concretamente la figura del Ministro de Defensa;

- Geopolítica: a partir de las misiones internacionales que España realiza en varios países y su papel en la OTAN;

- Modernización: tomando como referencia el papel de la mujer y su mayor integración en las Fuerzas Armadas;

- Responsabilidad: ante problemáticas internas.

Se ha seleccionado la técnica estadística de análisis multivariante, como es el análisis factorial, que permite tener medidas de un conjunto de variables y explicar las intercorrelaciones existentes entre ellas (Ferrando; Anguiano-Carrasco, 2010, p. 18). En cuanto a la realización del análisis factorial, se han seguido los procedimientos propuestos por Comrey (1985, p. 18):

a) seleccionar las variables;

b) calcular la matriz de correlaciones entre variables;

c) extraer los factores rotados;

d) rotar los factores;

e) interpretar la matriz de factores rotados.

Para calcular la matriz de correlaciones se ha utilizado el coeficiente de correlación de Pearson, aunque hay que advertir la disparidad de opiniones al respecto. Si bien es cierto que el análisis multivariante presenta dificultades a la hora de analizar variables dicotómicas, la necesidad de reducción de datos es una realidad apremiante en ciencias sociales. Autores como Kubinger (2003) señalan la necesidad de aplicar la correlación tetracórica, función no disponible en SPSS, en vez de la de Pearson, ya que da resultados más óptimos en cuanto a las magnitudes de las estimaciones (Hoffman et al., 2013). No obstante, investigadores como Bartholomew (1980) no consideran la utilización del coeficiente de correlación de Pearson como un elemento tan problemático ya que las estimaciones obtenidas a partir de variables con distribución asimétrica no son severamente alteradas si los valores del índice de ajuste root mean square error (RMSEA) se mantienen dentro de los estándares aceptados $(0$ - 0,08) (Jöreskog, 2001).

Como marca la bibliografía metodológica existente, existen dos tipos de análisis factoriales:

- análisis factorial exploratorio;

- análisis factorial confirmatorio.

Esta investigación se basa en un análisis factorial confirmatorio. Su justificación la encontramos en la posibilidad de establecer una hipótesis teóricamente consistente y reproducible, y la flexibilidad de estimar las correlaciones entre los factores. La utilidad de esta técnica, como señala Ferrán-Aranaz, radica en que:

"pretende pasar de un número elevado de variables, a un número más pequeño de elementos explicativos, los factores, que le permiten explicar de una manera más sencilla esa realidad” (Ferrán-Aranaz, 1996, p. 12).

Posteriormente, para determinar que este análisis es el adecuado, se han llevado a cabo:

- el test de esfericidad de Bartlett, que permite detectar la presencia de relación entre variables;

- la medida Kaiser-Meyer-Olkin (KMO) de adecuación de la muestra, para comparar las magnitudes de los coeficientes de correlación.

Con el fin de dar resultados más concretos, se ha procedido a realizar una rotación ortogonal varimax que permite la redistribución de la varianza de factores (Kaiser, 1958), facilitando así mostrar cuáles son los factores que presentan correlaciones muy altas y cuáles presentan correlaciones nulas. La elección de este tipo de rotación se debe a su amplia utilización en ciencias sociales y su fácil aplicación. De ahí que

"una de las razones por las que muchos investigadores prefieren las rotaciones ortogonales es que éstas son mucho más fáciles de comprender y simples de calcular que las soluciones oblicuas" (Comrey, 1985, p. 31). 
En definitiva, esta investigación sigue los pasos de otros estudios dedicados a la localización de encuadres, como son los análisis elaborados por D’Haenens y De-Langue (2001) o Setmeko y Valkenburg (2000).

Para facilitar al lector la comprensión de las variables, las categorías de respuesta de todas ellas constan de dos opciones: $0=$ No y 1 =Sí. A nuestro juicio, es la mejor forma de simplificar nuestro análisis y otorgarle cierta fiabilidad ya que, debido a las limitaciones de un trabajo de este tipo, no se puede contar con varios codificadores para eliminar el sesgo del investigador; de ahí la realización de preguntas dicotómicas para confirmar los encuadres con la técnica estadística propuesta.

Para la realización de los análisis se ha empleado el paquete estadístico SPSS versión 20.0.

\section{Análisis de resultados}

La tabla 1 muestra el análisis descriptivo de las variables utilizadas en el libro de codificación y su peso en los editoriales analizados. Atendiendo a los datos extraídos podemos inferir que en ambos períodos analizados el peso de los partidos políticos en el ámbito de las Fuerzas Armadas (FAS) ha sido considerable dentro de las rutinas periodísticas de los diarios españoles. De ahí que las menciones al ministro de Defensa presenten unas frecuencias significativas, aunque como se mostrará posteriormente esto se debe a ciertas peculiaridades.

Tabla 1. Variables y frecuencias en los periodos analizados

\begin{tabular}{|c|c|c|c|c|c|c|}
\hline \multirow{2}{*}{$\begin{array}{ll} & \text { Framing items } \\
\text { Período (2017-2018) } & \end{array}$} & \multicolumn{2}{|c|}{ Elpaís } & \multicolumn{2}{|c|}{ El mundo } & \multicolumn{2}{|c|}{ La vanguardia } \\
\hline & $\mathbf{n}$ & $\%$ & $\mathbf{n}$ & $\%$ & $\mathbf{n}$ & $\%$ \\
\hline ¿Se hace alguna mención al pasado franquista? & 5 & 17,24 & 0 & 0,00 & 0 & 0,0 \\
\hline ¿Aparece en el editorial algún partido político? & 17 & 58,62 & 10 & 35,71 & 8 & 47,05 \\
\hline ¿Se menciona a la OTAN? & 8 & 27,58 & 7 & 25,00 & 4 & 23,52 \\
\hline ¿Hace referencia a la modernización de las FAS? & 9 & 31,03 & 18 & 64,28 & 6 & 35,29 \\
\hline ¿Se menciona al ministro de Defensa? & 13 & 44,82 & 11 & 39,28 & 11 & 64,70 \\
\hline ¿Se hace referencia a alguna misión en la que participa España? & 14 & 48,27 & 14 & 50,00 & 6 & 35,29 \\
\hline ¿Se menciona algún grupo terrorista? & 10 & 34,48 & 6 & 21,42 & 6 & 35,29 \\
\hline ¿Hace referencia a algún tipo de acoso dentro del ejército? & 2 & 6,89 & 1 & 3,57 & 0 & 0,0 \\
\hline ¿Resalta el papel de la mujer en las FAS? & 6 & 20,68 & 10 & 35,71 & 2 & 11,76 \\
\hline ¿Menciona la relación entre España y EUA? & 4 & 13,79 & 6 & 21,42 & 6 & 35,29 \\
\hline Período (2012-2013) & $\mathbf{n}$ & $\%$ & $\mathbf{n}$ & $\%$ & $\mathbf{n}$ & $\%$ \\
\hline ¿Se hace alguna mención al pasado franquista? & 8 & 36,36 & 3 & 5,35 & 1 & 5,88 \\
\hline ¿Aparece en el editorial algún partido político? & 5 & 22,72 & 17 & 30,35 & 7 & 41,17 \\
\hline ¿Se menciona a la OTAN? & 5 & 22,72 & 20 & 35,71 & 4 & 23,52 \\
\hline ¿Hace referencia a la modernización de las FAS? & 7 & 31,91 & 21 & 37,50 & 6 & 35,29 \\
\hline ¿Se menciona al ministro de Defensa? & 6 & 27,27 & 29 & 51,78 & 10 & 58,82 \\
\hline ¿Se hace referencia a alguna misión en la que participa España? & 5 & 22,72 & 16 & 28,57 & 3 & 17,64 \\
\hline ¿Se menciona algún grupo terrorista? & 2 & 9,09 & 6 & 10,71 & 0 & 0,00 \\
\hline ¿Hace referencia a algún tipo de acoso dentro del ejército? & 2 & 9,09 & 1 & 1,78 & 1 & 5,88 \\
\hline ¿Resalta el papel de la mujer en las FAS? & 4 & 18,18 & 4 & 7,14 & 1 & 5,88 \\
\hline ¿Menciona la relación entre España y EUA? & 4 & 18,18 & 12 & 21,42 & 1 & 5,88 \\
\hline
\end{tabular}

Como se puede observar en la tabla 2, se ha llevado a cabo la prueba Kaiser-Meyer-Olkin (KMO), que ha permitido comparar las magnitudes de los coeficientes de correlación, y la prueba de esfericidad de Bartlett, para comprobar que nuestra matriz de datos corresponde a una matriz de identidad. Los resultados obtenidos indican que el análisis factorial se ha podido realizar satisfactoriamente ya que el resultado en el índice de esfericidad de Bartlett señala un valor muy inferior al límite establecido de 0,05 , con un resultado de ,000, y la prueba KMO señala un valor muy próximo a 1 . Como señala Montoya-Suárez:

"Los valores KMO entre 0,5 y 1 indican que es apropiado aplicar el análisis factorial a la matriz de datos bajo estudio" (Montoya-Suárez, 2007, p. 284).

En el caso de nuestra matriz de datos, la prueba KMO arrojó un valor de ,621 lo que indica que la muestra seleccionada en esta investigación es apropiada para la realización de un análisis factorial.

Como indica la bibliografía metodológica consultada para este estudio, los métodos para extraer los factores iniciales de la matriz de correlación son muy diversos. En

Tabla 2. Prueba KMO y esfericidad de Bartlett 1

\begin{tabular}{|c|c|c|}
\hline \multicolumn{2}{|c|}{ Medida de adecuación muestral de Kaiser-Meyer-Olkin } & 621 \\
\hline \multirow{3}{*}{ Prueba de esfericidad de Bartlett } & Chi-cuadrado aproximado & 207,612 \\
\hline & gl & 55 \\
\hline & Sig. &, 000 \\
\hline
\end{tabular}


nuestro caso se ha optado por el método de extracción de componentes principales para conocer qué factor explica el mayor porcentaje de la varianza en nuestra matriz de correlación. El análisis de componentes principales, indica la necesidad de extraer 3 factores (aquellos que sean mayores de 1) que, en nuestro caso de estudio explican el 55,862\% de nuestra muestra. Posteriormente, debido a la complejidad de interpretar los factores extraídos inicialmente, se procedió a realizar una rotación varimax con el fin de obtener unos resultados que ayudasen a simplificar nuestra interpretación a la hora de identificar los encuadres periodísticos. El método de rotación y la selección del número de factores extraídos corresponden a dos cuestiones principales:

- las diferencias entre el resto de factores presentan porcentajes mínimos en relación con el total de la varianza explicada;

- las rotaciones ortogonales, en este caso concreto la varimax, son fáciles de aplicar y son ampliamente usadas en estudios como el aquí planteado.

Tabla 3. Matriz de componentes rotados 1

\begin{tabular}{|c|c|c|c|}
\hline \multirow{2}{*}{ Framing items } & \multicolumn{3}{|c|}{ Componentes (2017-2018) } \\
\hline & Geopolítica & Modernización & Acoso \\
\hline ¿Se hace alguna mención al pasado franquista? & 109 &,- 126 & 784 \\
\hline ¿Aparece en el editorial algún partido político? &,- 251 &,- 594 & 253 \\
\hline ¿Se menciona a la OTAN? & 836 &,- 033 & ,036 \\
\hline ¿Hace referencia a la modernización de las FAS? &,- 004 &, 803 &,- 043 \\
\hline ¿Se menciona al ministro de Defensa? &,- 307 &,- 113 & ,288 \\
\hline ¿Se hace referencia a alguna misión en la que participa España? & ,727 & ,297 &,- 006 \\
\hline ¿Se menciona algún grupo terrorista? & 833 &,- 067 &,- 094 \\
\hline ¿Hace referencia a algún tipo de acoso dentro del ejército? &,- 136 & 201 & ,700 \\
\hline ¿Resalta el papel de la mujer en las FAS? &, 020 & ,808 & ,269 \\
\hline ¿Menciona la relación entre España y EUA? & 767 & ,088 &,- 064 \\
\hline
\end{tabular}

En nuestra matriz de componentes rotados (tabla 3) se puede apreciar que los resultados obtenidos han permitido, gracias a este método, extraer datos concretos y claros al aproximar las cargas altas al valor 1 o -1 y las cargas bajas de la matriz no rotada al valor 0 . Podemos identificar que el primer factor estaría compuesto por 4 variables que presentan una fuerte correlación. El resto (compuesto por 2 variables en ambos casos) presenta valores significativos que explican el contenido de la matriz, aunque con relaciones más débiles. Por lo tanto podemos destacar que durante los últimos dos años la prensa española ha utilizado 3 encuadres periodísticos, a los que hemos denominado: Geopolítica, Modernización y Acoso.

La tabla 4 muestra que la cobertura Tabla 4. Encuadres localizados en los medios editoriales analizados (2017-2018) de la prensa española, cuando trata asuntos relacionados con las Fuerzas Armadas, se caracteriza por dos fenómenos:

- una clara tendencia informativa a detallar las operaciones que las Fuerzas Armadas realizan en países

Denominación del encuadre a partir de la denominación del problema

Las misiones internacionales de las FAS son parte de la geopolítica OTAN

La incorporación de la mujer es un elemento para la modernización de las FAS

Las prácticas preconstitucionales son la causa del acoso dentro de las FAS extranjeros (Líbano, Afganistán, Irak), enmarcando el rol que ejercen dentro de la OTAN, haciendo claras referencias a las relaciones con EUA y la lucha contra el terrorismo yihadista:

"El soldado que ondeó la bandera española en el Líbano" (El país, 24/12/2017);

- destacar la modernización del ejército con la progresiva incorporación y ascenso de la mujer dentro de las Fuerzas Armadas, identificando los casos de acoso como una causa de prácticas arcaicas preconstitucionales:

“España podría contar con la primera mujer general en sus Fuerzas Armadas a partir de julio de 2019” (El mundo, 30/10/2018),

"Ejército activa protocolo acoso y estudia el caso de la legionaria que faltó al desfile" (La vanguardia, 09/04/2018).

Como podemos observar en la tabla 5, las pruebas KMO y de esfericidad de Bartlett permiten realizar nuestro segundo análisis factorial con respecto al segundo período temporal estudiado. Con unos valores de , 592 y ,000, las pruebas indican la idoneidad de aplicar la técnica estadística propuesta.

Tabla 5. Prueba KMO y esfericidad de Bartlett 2
\begin{tabular}{|l|l|r|}
\hline Medida de adecuación muestral de Kaiser-Meyer-Olkin &, 592 \\
\hline \multirow{3}{*}{ Prueba de esfericidad de Bartlett } & Chi-cuadrado aproximado & 222,664 \\
\cline { 2 - 3 } & gl & 45 \\
\cline { 2 - 3 } & Sig. &, 000 \\
\hline
\end{tabular}


Tabla 6. Matriz de componentes rotados 2

\begin{tabular}{|c|c|c|c|c|}
\hline \multirow{2}{*}{ Framing items } & \multicolumn{4}{|c|}{ Componentes (2012-2013) } \\
\hline & Geopolítica & Acoso sexista & Política & Modernización \\
\hline ¿Se hace alguna mención al pasado franquista? &,- 210 & ,044 &,- 702 &,- 045 \\
\hline ¿Aparece en el editorial algún partido político? &,- 221 & 031 & 347 &,- 678 \\
\hline ¿Se menciona a la OTAN? &, 515 &,- 089 & ,484 & ,238 \\
\hline ¿Hace referencia a la modernización de las FAS? &,- 149 &,- 059 & 161 & ,854 \\
\hline ¿Se menciona al ministro de Defensa? &,- 263 &,- 138 & 711 &,- 209 \\
\hline ¿Se hace referencia a alguna misión en la que participa España? & 837 &,- 120 &,- 029 &,- 050 \\
\hline ¿Se menciona algún grupo terrorista? & 793 &,- 032 &,- 091 &,- 014 \\
\hline ¿Hace referencia a algún tipo de acoso dentro del ejército? &,- 032 & ,908 &,- 043 &,- 062 \\
\hline ¿Resalta el papel de la mujer en las FAS? &,- 137 &, 886 &,- 132 &,- 021 \\
\hline ¿Menciona la relación entre España y EUA? &, 828 &,- 036 & 164 & ,065 \\
\hline
\end{tabular}

Tras la realización de la rotación varimax (tabla 5) nuestra segunda matriz de componentes arroja la necesidad de extraer 4 factores, que en este caso explican un 68,882\% de nuestra segunda muestra, lo que refuerza aún más este segundo análisis. Como resultado de ello se han localizado 4 encuadres periodísticos, que hemos denominado, en la línea de lo anteriormente expresado, Geopolítica, Acoso sexista, Política y Modernización.

La cobertura sobre las Fuerzas Armadas ha experimentado fluctuaciones desde 2012 hasta la actualidad como se ha podido observar en las distintas tablas de este documento. En este período estudiado se ha detectado la presencia de cuatro encuadres. Si bien los encuadres se mantienen y siguen una misma línea mediática, sus causas y variables explicativas han experimentado cambios.

El encuadre Geopolítica sigue siendo el que presenta resultados estadísticos más sólidos, algo normal ya que las misiones que realiza España en el extranjero son el tema informativo por excelencia de las Fuerzas Armadas:

“El ejército español inaugura la sede de la gobernación afgana" (La vanguardia, 09/09/2013).

Los resultados más interesantes los encontramos en los encuadres Acoso sexista y Modernización. En el primero existe una fuerte correlación entre las funciones de las mujeres militares y los casos de acoso sexual dentro de la institución militar:

“62 militares se han quejado de acoso sexual en diez años según Defensa” (El país, 19/09/2013).

Por otro lado, la cobertura del segundo no está centrada en el rol de la mujer sino en cuestiones tecnológicas y económicas donde hay una intervención directa de los partidos políticos:

"El ejército del futuro, con más robots y menos soldados" (El país, 21/09/2012).

Finalmente se detectó la presencia de un cuarto encuadre, Política, cuya presencia entendemos que está fuertemente relacionada con la figura del exministro Pedro Morenés, debido a varias polémicas políticas referentes a cuestiones internas de las Fuerzas Armadas y externas, como la crisis de Cataluña:

"Morenés precisa que se refirió a las críticas por las maniobras en Cataluña" (El país, 08/01/2013);

“Morenés dice que España defenderá la ley con ‘todas las medidas que hagan falta'” (El mundo, 10/10/2013).

\section{Conclusiones}

Se puede apreciar que las mujeres que conforman las Fuerzas Armadas son un colectivo de una importante repercusión mediática en la prensa española cuando se cubren asuntos militares. A partir del análisis factorial de los dos períodos seleccionados, podemos confirmar lo que planteábamos en nuestra hipótesis de trabajo (H1). Debido a la inexistencia de trabajos que hayan tratado esta cuestión, y tomando como referencia que las cuestiones de defensa nacional no ocupan un lugar de suma importancia para la sociedad española, no sorprende ver que la muestra de este trabajo se considere reducida si lo comparamos
Mediáticamente la mujer militar ya no es una víctima sino un paso de modernización castrense 
con otros estudios de comunicación. Pero, debido a esa inexistencia, podemos afirmar que no sólo el encuadre de género se encuentra presente en la cobertura mediática, sino que ha experimentado modificaciones importantes, lo que demuestra el interés de este trabajo para la comunicación y la ciencia política.

\section{El encuadre Política es meramente co- yuntural por la repercusión del exminis- tro Pedro Morenés en los medios}

Partiendo de la base de los estudios que han tratado las Fuerzas Armadas y su relación con la opinión pública y los medios de comunicación, era de esperar que los encuadres Geopolítica y Política tuvieran mayor peso en nuestro estudio. Aunque en el caso del segundo se ha podido establecer una fuerte relación entre la actividad militar y los personalismos políticos de sus máximos referentes, como es la figura del ministro de Defensa. Sería interesante ahondar en esta cuestión en investigaciones futuras para conocer la influencia del liderazgo político dentro de las Fuerzas Armadas Españolas. Investigaciones que sí se han llevado a cabo fuera de España, como los estudios de Castro y Lupano (2007). No obstante, planteamos que la presencia del encuadre Política resulta coyuntural por las polémicas en las que estuvo envuelto el exministro Pedro Morenés.

La aportación principal de este estudio proviene principalmente de la cobertura sobre la modernización de las Fuerzas Armadas y qué factores integran esa modernización. Si bien hace años (2012-2013) el proceso de actualización del ejército se centraba en factores económicos y políticos, especialmente en el rejuvenecimiento de los instrumentos militares, en la actualidad el progreso y ascenso de las mujeres ocupa la cobertura mediática sobre esta cuestión. A pesar de que, como se ha demostrado en este estudio, el acoso sigue siendo un elemento altamente noticiable, éste ha sufrido una mutación. Ha pasado de ser considerado un fenómeno puramente sexual (2012-2013), a una acción persecutoria dentro del ejército y a ser mediáticamente identificado como una rémora de prácticas preconstitucionales.

Los últimos estudios del CIS han mostrado la presencia en la sociedad de una opinión pública favorable a la figura de las Fuerzas Armadas. Sin duda será interesante analizar, a partir de los datos de esta investigación, la cobertura sobre asuntos militares, donde el acoso sigue siendo un elemento importante, para comprobar si se mantiene o ha mermado la buena imagen pública de las Fuerzas Armadas, ya que el último estudio del CIS data de 2015.

En definitiva, este trabajo, a pesar de sus limitaciones, puede servir de base para futuras investigaciones que deseen ahondar en la relación entre las Fuerzas Armadas españolas y los medios de comunicación.

\section{Referencias}

Agudo-Arroyo, Yolanda (2014). "La participación de las mujeres en las Fuerzas Armadas españolas: de la incorporación a la integración". Comunitania: Revista internacional de trabajo social y ciencias sociales, n. 7, pp. 9-27. https://doi.org/10.5944/comunitania.7

Ardèvol-Abreu, Alberto (2015). “Framing o teoría del encuadre en comunicación. Orígenes, desarrollo y panorama actual en España". Revista latina de comunicación social, n. 70, pp. 423-450.

https://doi.org/10.4185/RLCS-2015-1053

Bartholomé, Guus; Lecheler, Sophie; De-Vreese, Claes (2018). "Towards a tipology of conflict frames. Substantiveness and interventionism in political conflict news". Journalism studies, v. 19, n. 12, pp. 1-23.

https://doi.org/10.1080/1461670X.2017.1299033

Bartholomew, David (1980). "Factor analysis for categorical data”. Journal of the Royal Statistical Society. Series B (Methodological), v. 42, n. 3, pp. 293-321.

https://www.jstor.org/stable/2985165

Bennett, W. Lance (1990). "Toward a theory of press-state relations in the United States". Journal of communication, v. 40, n. 2, pp. 103-127.

https://doi.org/10.1111/j.1460-2466.1990.tb02265.x

Berríos, Paulina (2018). "El sistema de prestigio en las universidades y el rol que ocupan las mujeres en el ámbito académico". Calidad en la educación, n. 23, pp. 349-361.

https://doi.org/10.31619/caledu.n23.301

Castro, Alejandro; Lupano, María-Laura (2007). “Teorías implícitas del liderazgo y calidad de la relación entre líder y seguidor". Boletín de psicología, n. 89, pp. 7-28.

https://www.uv.es/seoane/boletin/previos/N89-1.pdf

Comrey, Andrew L. (1985). Manual de análisis factorial. Madrid: Cátedra. ISBN: 9788437605159

Coocky, Cheryl (2018). "Gender, sport and media between the mid-1980s and early 2000s: Developments trajectories and transformations". In: Mansfield, Louise; Caudwell, Jayne; Wheaton, Belinda; Watson, Brandon (eds.). The palgrave handbook of feminism and sport, leisure and physical education. London: Palgrave Macmillan, pp. 133-147. ISBN: 9781 137533173 
https://doi.org/10.1057/978-1-137-53318-0_9

DeCillia, Brooks (2018). “'But it is not getting any safer!': The contested dynamic of framing Canada's military mission in Afghanistan". Canadian journal of political science, v. 51, n. 1, pp. 155-177.

https://doi.org/10.1017/S0008423917000634

D’Haenens, Leen; De-Langue, Marielle (2001). "Framing of asylum seekers in Dutch regional newspapers". Media culture \& society, v. 23, n. 6, pp. 847-860.

https://doi.org/10.1177/016344301023006009

Díez-Nicolás, Juan (1986). “La transición política y la opinión pública española ante los problemas de la defensa y las Fuerzas Armadas". Revista española de investigaciones sociológicas, n. 36, pp. 13-24.

https://doi.org/10.2307/40183243

Entman, Robert (1993). “Framing: Toward clarification of a fractured paradigm". Journal of communication, v. 43, n. 4, pp. 51-58.

https://doi.org/10.1111/j.1460-2466.1993.tb01304.x

Entman, Robert (2004). Projections of power: Framing news, public opinion, and US foreign policy. Chicago: Chicago University Press. ISBN: 9780226210728

Fernández-Vargas, Valentina (1997). Las militares españolas: un nuevo grupo profesional. Madrid: Biblioteca Nueva. ISBN: 9788470305139

Ferrán-Aranaz, Magdalena (1996). "SPSS para Windows 95”. Programación y análisis estadístico. Madrid: McGraw-Hill. ISBN: 9788448105891

Ferrando, Pere-Joan; Anguiano-Carrasco, Cristina (2010). "El análisis factorial como técnica de investigación social". Papeles del psicólogo, v. 31, n. 1.

http://www.redalyc.org/html/778/77812441003

García-Marín, Javier (2011). “Conflictos y cobertura mediática: una aproximación desde la comunicación política”. Cuadernos de estrategia, n. 148, pp. 97-128.

https://dialnet.unirioja.es/servlet/articulo?codigo $=3838704$

Hoffman-Freiberg, Agustín; Stover, Juliana-Beatriz; De-la-Iglesia, Guadalupe; Fernández-Liporace, Mercedes (2013). "Correlaciones policóricas y tetracóricas en estudios factoriales exploratorios y confirmatorios". Ciencias psicológicas, v. 7, n. 2, pp. 151-164.

http://www.scielo.edu.uy/scielo.php?\%20pid=S1688-42212013000200005\&script=sci_arttext\&t/ng=en

Hombrados, Angustias; Olmedo, José; Val, Consuelo (2007). La incorporación de las mujeres a las Fuerzas Armadas: el caso español y su percepción en la opinión pública en perspectiva comparada. Madrid: Real Instituto Elcano de Estudios Internacionales y Estratégicos. Documentos de trabajo, n. 7.

Jordán, Javier; Torres, Manuel R. (2004). "La comunicación política en tiempos de guerra”. Revista general de Marina, pp. 589-598.

https://www.upo.es/export/portal/com/bin/portal/upo/profesores/mrtorsor/profesor/1214214230858_la_comunicacixn_ polxtica_en_tiempos_de_guerra-rgm.pdf

Jöreskog, Karl G. (2001). Analysis of ordinal variables 2. Cross-sectional data. Taller "Structural Equation Modelling with Lisrel 8.51. Friedrich-Schiller-Universitat, Jena.

Kaiser, Henry F. (1958). "The varimax criterion of analytic rotation in factor analysis". Psychometrika, v. 23, n. 3, pp. 187200.

https://doi.org/10.1007/BF02289233

Kubinger, Klaus (2003). "On artificial results due to using factor analysis for dichotomous variables". Psychology science, v. 45, n. 1 , pp. $106-110$.

http://citeseerx.ist.psu.edu/viewdoc/download?doi=10.1.1.495.778\&rep=rep1\&type=pdf

Kuhl, Steven; Kosloski, Anna E.; Ryon, Sthepanie-Bontranger; Monar, Astrid (2018). "Masculinity, organizational culture, media framing and sexual violence in the military". Social sciences, v. 7, n. 5, pp. 80.

https://doi.org/10.3390/socsci7050080

Lambert, Cheryl-Ann (2018). "A media framing analysis of a US presidential advisor: Alternative flacks". Public relations review, v. 44, n. 5, pp. 724-733.

https://doi.org/10.1016/j.pubrev.2018.07.006

León, Magdalena (2001). "El empoderamiento de las mujeres: encuentro del primer y tercer mundos en los estudios de género". Revista de estudios de género: La ventana, v. 2, n. 13, pp. 94-106. 
http://revistalaventana.cucsh.udg.mx/index.php/LV/article/view/553

Montoya-Suárez, Omar (2007). “Aplicación del análisis factorial a la investigación de mercados. Caso de estudio". Scentia et technica, v. 1, n. 35.

https://revistas.utp.edu.co/index.php/revistaciencia/article/view/5443

Muñiz, Carlos (2011). "Encuadres noticiosos sobre migración en la prensa digital mexicana: Un análisis de contenido exploratorio desde la teoría del framing". Convergencia, v. 18, n. 55, pp. 213-239.

http://www.redalyc.org/pdf/105/10515210009.pdf

Nicolini, Kristine M.; Hansen, Sara-Steffes (2018). “Framing the women's March on Washington: Media coverage and organizational messaging alignment. Public relations review, v. 44, n. 1, pp. 1-10.

https://doi.org/10.1016/j.pubrev.2017.12.005

Pizarroso-Quintero, Alejandro (2000). “Comunicación y defensa en la Europa contemporánea”. En: Modelo de Seguridad y Defensa en Europa en el próximo siglo. X Jornadas Universidad Complutense de Madrid - Ceseden. Madrid: Ministerio de Defensa, pp. 43-62. ISBN: 8478237585

https://dialnet.unirioja.es/servlet/libro?codigo $=562768$

Ricoy-Casas, Rosa-María (2006). "La difícil presencia de la mujer en las Fuerzas Armadas: de la mera prohibición al techo de cristal para su promoción y ascenso en un mundo tradicionalmente masculino". Dossiers feministes, n. 9, pp. 225-243. http://www.e-revistes.uji.es/index.php/dossiers/article/view/711

Rodríguez-Esperanza, María-Soledad; Humanes, María-Luisa (2017). “El conflicto palestino-israelí en la prensa española: la cobertura de la Operación Margen Protector en ABC y La vanguardia”. Observatorio, v. 11, n. 4, pp. $154-180$. https://doi.org/10.15847/obsOBS11420171136

Schattschneider, Elmer (1975). The semi-sovereign people: A realist's view of democracy in America. Belmont: Wadsworth Publishing. ISBN: 9780030133664

Setmeko, Holli A.; Valkenburg, Patti M. (2000). "Framing European politics: A content analysis of press and television news". Journal of communication, v. 50, n. 2, pp. 93-109.

https://doi.org/10.1111/j.1460-2466.2000.tb02843.x

Solana-Cortés, Manuel (2009). “El reclutamiento en las Fuerzas Armadas Españolas. Un caso singular: los primeros 20 años de presencia de la mujer". En: Gómez-Escarda, María; Sepúlveda-Muñoz, Isidro (eds.). Las mujeres militares en España (1988-2008). Madrid: Instituto General Gutiérrez Mellado, pp. 121-156. ISBN: 9788460809128 https://iugm.es/wp-content/uploads/2016/07/mujeres_miltares_Espana.pdf

Telcan-Reyes, Ana-Belén (2018). Estudio de la agenda-setting en el tratamiento de notas informativas periodísticas protagonizadas por mujeres, en diarios de circulación provincial. Machala: Universidad Académica de Ciencias Sociales. http://repositorio.utmachala.edu.ec/handle/48000/12193

Vicent, Richard C. (2000). "A narrative analysis of US press coverage of Slobodan Milosevic and the Serbs in Kosovo". European journal of communication, v. 15, n. 3, pp. 321-344.

https://doi.org/10.1177/0267323100015003004

\section{Cronología de la Documentación Española}

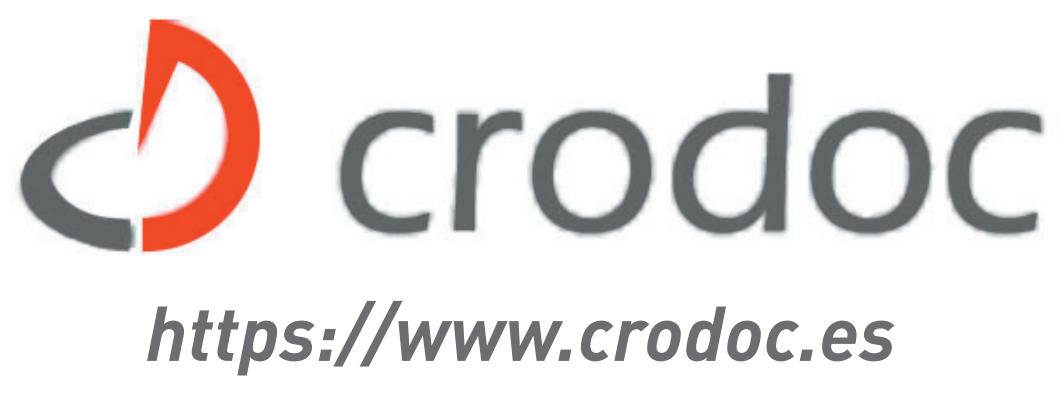

